\title{
COMPLETENESS OF REGULAR INDUCTIVE LIMITS
}

\section{JAN KUCERA and KELLY MCKENNON}

\author{
Department of Mathematics \\ Washington State University \\ Pullman, WA 99164 \\ (Received September 5, 1987)
}

ABSTRACT. Regular LB-space is fast complete but may not be quasi-complete. Regular inductive limit of a sequence of fast complete, resp. weakly quasi-complete, resp. reflexive Banach, spaces is fast complete, resp. weakly quasi-complete, resp. reflexive complete, space.

KEY WORDS AND PHRASES. Regular locally convex inductive limit, complete, quasi-complete, fast complete space.

1980 AMS SUBJECT CLASSIFICATION CODES. Primary 46M40, Secondary 46 A12.

1. INTRODUCTION.

In $[1, \S 31.6]$ Kothe has a sequence of Banach spaces $E_{1} \subset E_{2} \subset \ldots$ whose inductive limit is not quasi-complete. In [2] there is an example of reflexive Frechet spaces $E_{n}$ whose inductive limit is not even fast complete. Since an LF-space is fast complete iff it is regular, see [3], there is a natural question asked by Jorge Mujica in [4]: Is every regular $\mathrm{LB}-\mathrm{space}$ complete?

Throughout the paper $E_{1} \subset E_{2} \subset \ldots$ is a sequence of locally convex spaces with continuous inclusions $\mathrm{E}_{\mathrm{n}} \rightarrow \mathrm{E}_{\mathrm{n}+1}, \mathrm{n} \varepsilon \mathrm{N}$. Their locally convex inductive limit is denoted by $\mathrm{E}$. The space $\mathrm{E}$ is called regular if every set bounded in $\mathrm{E}$ is bounded in some $\mathrm{E}_{\mathrm{n}}$.

\section{MAIN RESULTS.}

Let $F$ be a locally convex space and $A \subset F$ absolutely convex. We denote by $F_{A}$ the seminormed space $U\{n A ; n \in N\}$ whose topology is generated by the Minkowski functional of $A$. If $F_{A}$ is Banach space, $A$ is called Banach disk. The space $F$ is called fast complete if every set bounded in $F$ is contained in a bounded Banach disk. Every sequentially complete space is fast complete and there are fast complete spaces which are sequentially incomplete, see [5].

EXAMPLE. For each $\mathrm{n} \varepsilon \mathrm{N}$ and $\mathrm{x}: \mathrm{NxN} \rightarrow \mathrm{C}$, put

$$
\begin{gathered}
\|x\|_{n}=\max \left\{\sup \left\{j^{-i}\left|x_{i j}\right| ; i \leqq n, j \varepsilon N\right\}, \sup \left\{\left|x_{i j}\right| ; i>n, j \varepsilon N\right\}\right\}, \\
E_{n}=\left\{x ;\|x\|_{n}<+\infty \& \lim { }_{j \rightarrow \infty} x_{i j}=0 \text { for } i>n\right\}, B_{n}=\left\{x \varepsilon E_{n} ;\|x\|_{n} \leqq 1\right\}, \text { an: }
\end{gathered}
$$


$E=$ indlim $E_{n}$. We prove that each $E_{n}$ is a Banach space, $E_{1} \subset E_{2} \subset \ldots$, inclusions $E_{n} \rightarrow E_{n+1}, n \in N$, are continuous, $E$ is regular and not quasi-complete.

CLAIM 1. Each space $\mathrm{E}_{\mathrm{n}}$ is Banach.

PROOF. Let $\{x(k)\}$ be a Cauchy sequence in $E_{n}$. For each $i, j \varepsilon N$ the sequence $\left\{x(k)_{i j}\right\}$ is Cauchy in $C$ and has a limit $x_{i j}$. Let $x$ be the matrix with the entries $x_{i j}$. Given $\varepsilon>0$, there is $k$ such that $p, r \geqq k$ implies $\|x(p)-x(r)\|_{n} \leq \varepsilon$. Hence, $\|x(p)-x\|_{n} \leqq \lim \sup _{r \rightarrow \infty}\|x(p)-x(r)\|_{n} \leqq \varepsilon$ and $\|x\|_{n} \leqq\|x-x(p)\|_{n}+\|x(p)\|_{n}<+\infty$.

Take $i>n$ and choose $j_{i}$ so that $\left|x(k)_{i j}\right|<\varepsilon$ for $j>j_{i}$. Then $\left|x_{i j}\right| \leqq\left|x_{i j}-x(k)_{i j}\right|+\left|x(k)_{i j}\right| \leqq\|x-x(k)\|_{n}+\left|x(k)_{i j}\right|<2 \varepsilon$ and $\lim _{j \rightarrow \infty} x_{i j}=0$.

CLAIM 2. $E_{1} \subset E_{2} \subset \ldots$ and each inclusion $E_{n} \rightarrow E_{n+1}$ is continuous. Proof follows from the inequalities $\|x\|_{1} \geqq\|x\|_{2} \geqq \ldots, x \in U\left\{E_{n} ; n \varepsilon N\right\}$.

CLAIM 3. E is regular.

PROOF. Let $D \subset E$ be not bounded in any $E_{n}$. For each $n \in N$ choose $x(n) \varepsilon D$ such that $\|x(n)\|_{n}>n$. There are $i(n), j(n) \in N$ for which

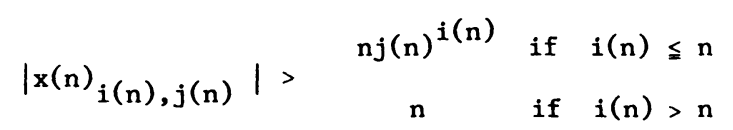

Put $m(n)=n+\max \{i(k) ; k \leqq n\}$ and $r(n)=\min \left\{j(k)^{-i(k)} ; k \leq m(n)\right\}, n \varepsilon N$.

If $k>n$ then $\|x(n)\|_{m(k)} \geq\left.\right|_{m(k)} \geq\left|j(n)^{-i(n)} x(n){ }_{i(n) j(n)}\right|>n r(k)$, if $k \leqq n$ then $\|x(n)\|_{m(k)} \geqq\|x(n)\|_{m(n)}>n r(k)$.

Let $V=U\left\{r(k) B_{m(k)} ; k \in N\right\}$ and $U=\operatorname{coV}$. Assume $x(n) \varepsilon n U$. Then

$$
x(n)=\sum_{k=1}^{s} \alpha_{k} y(k)
$$

where $\alpha_{k} \geqq 0, \Sigma \alpha_{k}=1$, and $y(k) \varepsilon n r(k) B_{m(k)}$. To prove that $\left|y(k){ }_{i(n) j(n)}\right| \leqq n$ for $k \in N$, we have to distinguish three cases:

(a) $k>n$ : Then $\left|y(k){ }_{i(n), j(n)}\right|=\left|y(k){ }_{i(n), j(n)} j(n)^{i(n)-i(n)}\right| \leqq$

$$
\leqq\|y(k)\|_{m(k)} j(n)^{i(n)} \leqq n r(k) j(n)^{i(n)} \leqq n .
$$


(b) $k \leq n \& i(n) \leqq m(k)$ : Then $\left|y(k)_{i(n), j(n)}\right| \leqq \mid y(k) \|_{m(k)} j(n)^{i(n)} \leq$ $\leqq n r(k) j(n)^{i(n)} \leqq n$.

(c) $k \leqq n \& i(n)>m(k)$ : Then $\left|y(k)_{i(n), j(n)}\right| \leq\|y(k)\|_{m(k)} \leq n r(k) \leqq n$.

On the other hand $|x(n) i(n), j(n)|>n$ and $x(n)$ cannot be a convex combination of $y(k), k \leq s$, i.e. $x(n) \notin n U$. Since $U$ is a 0 -neighborhood in E, $D$ is not bounded in E.

CLAIM 4. E is not quasi-complete.

PROOF. Let $\Delta=\{\delta \subset N x N ;\{j \varepsilon N ;(i, j) \varepsilon \delta\}$ is finite, $i \varepsilon N\}$ be ordered by set inclusion. Denote by $x(\delta)$ the set characteristic function of $\delta \varepsilon \Delta$. Then $\{x(\delta) ; \delta \varepsilon \Delta\}$ $\subset \mathrm{B}_{1}$ and the filter associated with $\delta \rightarrow \mathrm{x}(\delta)$ is bounded in $\mathrm{E}_{1}$, hence also bounded in $\mathrm{E}$.

Let $P_{n}: C^{N x N} \rightarrow C^{N x N}$ be the projection of an $N x N$ matrix on its $n-t h$ row. Take a a closed absolutely convex 0 -neighborhood $V$ in $E$. For each $n \in N$ choose $m(n) \varepsilon N$ and $r(n)>0$ so that $r(n) B_{n} \subset v, m(n) \geq 2 r(n)^{-1 / n}$, and put $\sigma=\{(i, j) \varepsilon N x N j \leq m(i)\}$. If $\gamma, \delta \varepsilon \Delta, \gamma, \delta \geq \sigma$, then $x(\gamma)_{i j}-x(\delta)_{i j}=0$ for $j \leq m(i)$ and $\left\|P_{n}(x(\gamma)-x(\delta))\right\|_{n}=$ $\sup \left\{j^{-n}\left|x(\gamma)_{n j}-x(\delta)_{n j}\right| ; j>m(n)\right\}<m(n)^{-n} \leq 2^{-n} r(n)$. Hence $2^{n_{p}} P_{n}(x(\gamma)-x(\delta))$ $\varepsilon r(n) B_{n} \subset V$. Since $V$ is absolutely convex, the sequence

$$
y_{k}=\sum_{n=1}^{k} 2^{-n} 2^{+n} P_{n}(x(\gamma)-x(\delta)), k \varepsilon N
$$

is contained in $V$. It is also contained in $B_{1}$ and converges coordinate-wise to $x(\gamma)-x(\delta)$ in $E_{1}$. Hence $x(\gamma)-x(\delta)$ is in the weak closure of $V$. Since $V$ is closed and convex, it is also weakly closed and $x(\gamma)-x(\delta) \varepsilon V$. So $\{x(\delta) ; \delta \varepsilon \Delta\}$ is a base of a bounded Cauchy filter in $E$. If it had a limit $x \varepsilon E$, then $x_{i j}=1$ for all $i, j \varepsilon N$. This would imply $x \notin E_{n}$ for any $n \varepsilon N$ and $x \notin E$, q.e.d.

LEMMA. Regular inductive limit of a sequence of semireflexive, resp. reflexive, spaces is semireflexive, resp. reflexive.

PROOF. Let each $E_{n}$ be semireflexive. Since $E=$ indlim $E_{n}$ is regular, its strong dual $E_{b}^{\prime}$ equals to projlim $\left(E_{n}\right)_{b}^{\prime}$ and $\left(E_{b}^{\prime}\right)^{\prime} \subset U\left\{\left(\left(E_{n}\right)_{b}^{\prime}\right)^{\prime} ; n \varepsilon N\right\}=U\left\{E_{n} ; n \varepsilon N\right\}=E$.

Let each $E_{n}$ be reflexive. By [7;IV, 5.6] it suffices to show that $E$ is semireflexive and barreled. Take a barrel $B$ in $E$. For each $n \varepsilon N, B \cap E_{n}$ is a barrel in $E_{n}$. Since $E_{n}$ is reflexive, the barrel $B \cap E_{n}$ is a neighborhood in $E_{n}$, which implies that $B$ is a neighborhood in $\mathrm{E}$ and $\mathrm{E}$ is barreled.

CONSEQUENCE. Inductive limit of a sequence of reflexive Banach spaces is reflexive.

PROOF. By [6; Th. 4] the inductive limit of reflexive Banach spaces is regular. 
THEOREM. Let $E=$ indlim $E_{n}$ be regular. Then:

(a) Each $E_{n}$ fast complete $\Rightarrow \mathrm{E}$ fast complete.

(b) Each $E_{n}$ weakly quasi-complete $\Rightarrow E$ weakly quasi-complete.

(c) Each $E_{n}$ semireflexive $\Rightarrow E$ quasi-complete.

(d) Each $E_{n}$ reflexive Banach $\Rightarrow E$ complete.

PROOF.

(a) Let $B \subset E$ be bounded, then it is bounded in some $E_{n}$ and contained in a bounded Banach disk in $E_{n}$. Since any Banach disk bounded in $E_{n}$ is also bounded in $E$, the proof is complete.

(b) Follows from Lemma since any locally convex space is weakly quasi-complete iff it is semireflexive, $[7 ; \mathrm{IV}, 5.5]$.

(c) Follows from (b) since every weakly quasi-complete space is quasi-complete.

(d) Let $F$ be a Cauchy filter in E. Then $F$, as a filter of continuous linear functionals on $E_{b}^{\prime}$, converges uniformly on bounded sets in $E_{b}^{\prime}$ to $a$ linear, not necessarily continuous, functional $h: E_{b}^{\prime} \rightarrow C$. Since $E$ is reflexive, it suffices to show that $h$ is continuous.

$h$ is continuous iff $h^{-1}(0)$ is closed in $E_{b}^{\prime}$. The space $E$ is regular, [6; Th. 4], hence $E_{b}^{\prime}=$ projlim $E_{n}^{\prime}$ is Frechet. Take a sequence $\left\{x_{n} ; n=1,2 \ldots\right\} \subset h^{-1}(0)$ which converges to $x_{0}$ in $E_{b}^{\prime}$. We have to show that $h\left(x_{0}\right)=0$. Choose $\varepsilon>0$. The set $B=\left\{x_{n} ; n=0,1,2, \ldots\right\}$ is bounded in $E_{b}^{\prime}$, hence there is $F \varepsilon F$ such that $\sup \left\{\left|f\left(x_{n}\right)-h\left(x_{n}\right)\right| ; f \varepsilon F, x_{n} \varepsilon B\right\}<\varepsilon$. Fix an $f \varepsilon F$ and choose $n \varepsilon N$ so that $\left|f\left(x_{n}\right)-f\left(x_{0}\right)\right|<\varepsilon$. Then $\left|h\left(x_{0}\right)\right|=\left|h\left(x_{0}\right)-h\left(x_{n}\right)\right| \leq\left|h\left(x_{0}\right)-f\left(x_{0}\right)\right|+$ $\left|f\left(x_{0}\right)-f\left(x_{n}\right)\right|+\left|f\left(x_{n}\right)-h\left(x_{n}\right)\right|<3 \varepsilon$, which implies $h\left(x_{0}\right)=0$.

CONJECTURE. Regular LB-space may not be sequentially complete.

\section{REPERENCES}

1. KOTHE, G. Topological vector spaces I, Springer Verlag, 1969.

2. KUCERA, J., MCKENNON, K. Kothe's example of an incomplete LB-space, Proc. Amer. Math. Soc., Vol. 93, No. 1, (1985), 79-80.

3. KUCERA, J., BOSCH, C. Bounded sets in fast complete inductive limits, Int. J. Math.\& Math. Sci., Vol. 7, No. 3, (1984), 615-617.

4. MUJICA, J. Functional analysis, holomorphy and approximation theory II, North Holland 1984 .

5. BOSCH, C., KUCERA, J., MCKENNON, K. Fast complete locally convex linear topological spaces, Internat. J. Math. \& Math. Sci. Vol. 9, No. 4, (1986), 791-796.

6. KUCERA, J., MCKENNON, K. Dieudonne-Schwartz theorem on bounded sets in inductive limits, Proc. Amer. Math. Soc., Vol. 78, No. 3, (1980), 366-368.

7. SCHAEFER, H. Topological vector spaces, Springer Verlag, 1971. 


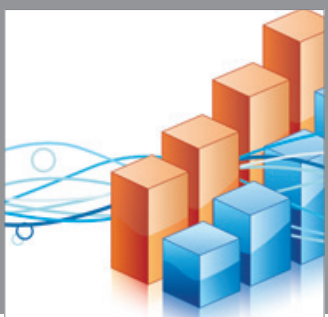

Advances in

Operations Research

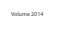

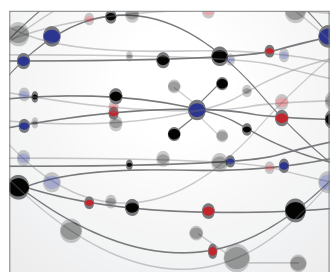

\section{The Scientific} World Journal
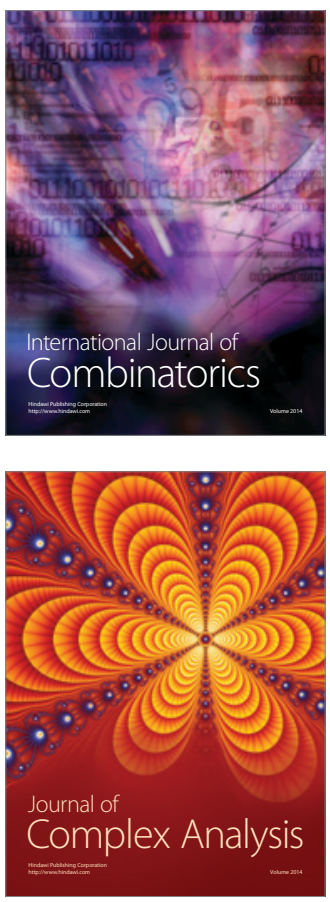

International Journal of

Mathematics and

Mathematical

Sciences
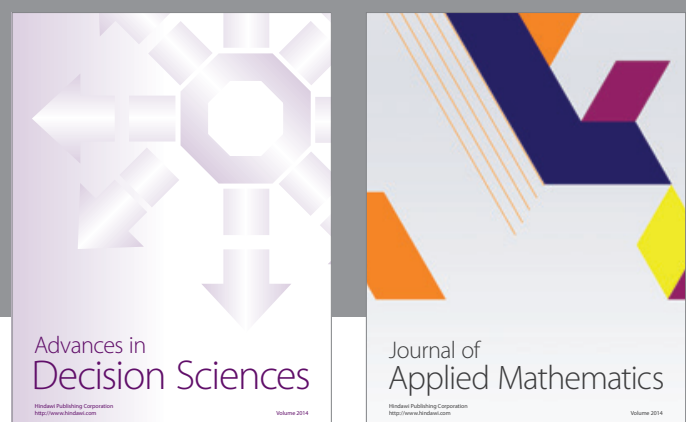

Journal of

Applied Mathematics
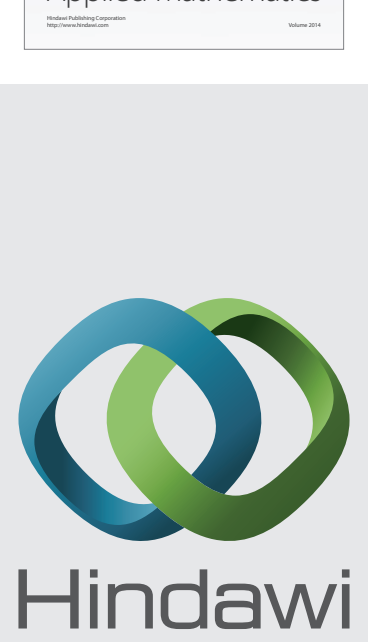

Submit your manuscripts at http://www.hindawi.com
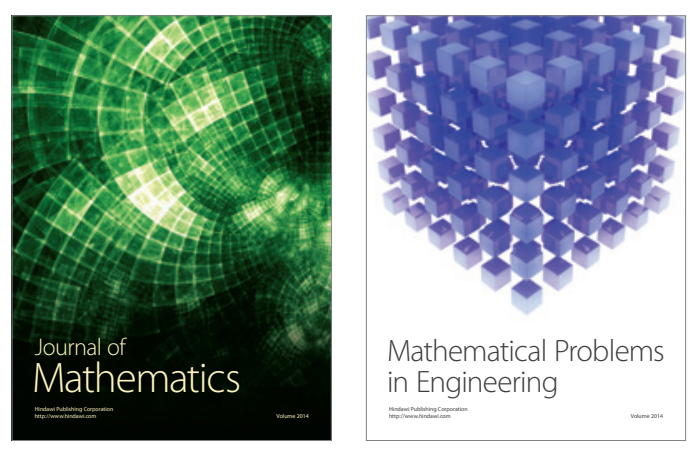

Mathematical Problems in Engineering
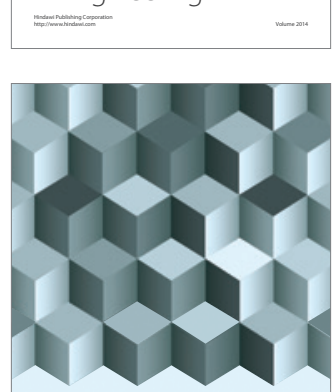

Journal of

Function Spaces
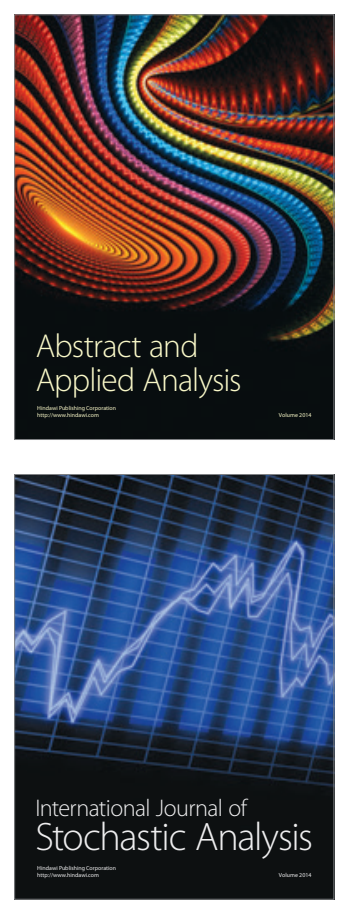

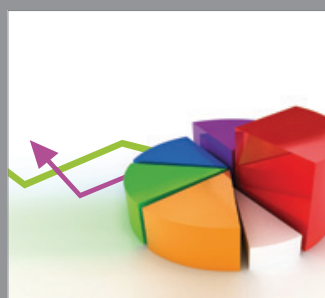

ournal of

Probability and Statistics

Promensencen
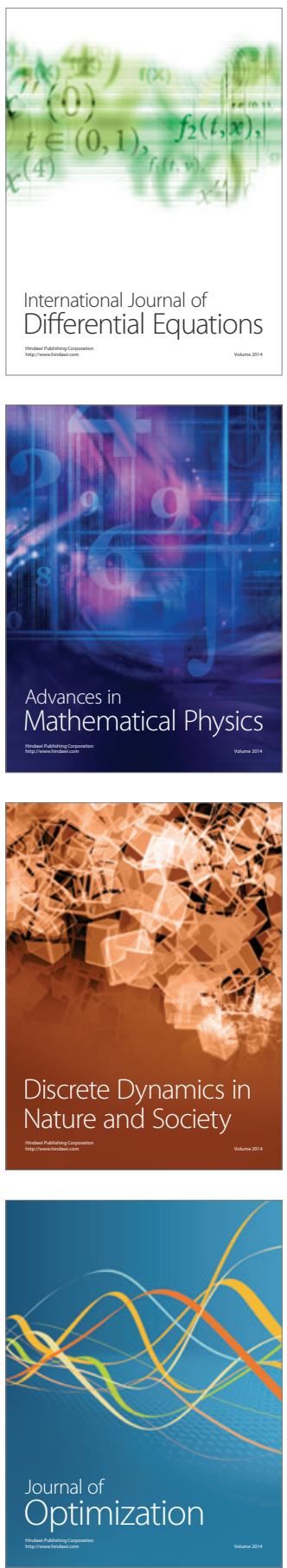